\title{
Linkage of Heterogeneous Knowledge Resources within In-Store Dialogue Interaction
}

\author{
Sabine Janzen ${ }^{1}$, Tobias Kowatsch ${ }^{2}$, Wolfgang Maass ${ }^{1,2}$, and Andreas Filler ${ }^{1}$ \\ 1 Furtwangen University, Robert-Gerwig-Platz 1, 78120 Furtwangen, Germany \\ \{sabine.janzen, wolfgang.maass, andreas.filler\}@hs-furtwangen.de \\ 2 University of St. Gallen, Dufourstrasse 40a, 9000 St. Gallen, Switzerland \\ \{tobias.kowatsch, wolfgang.maass\}@unisg.ch
}

\begin{abstract}
Dialogue interaction between customers and products improves presentation of relevant product information in in-store shopping situations. Thus, information needs of customers can be addressed more intuitive. In this article, we describe how access to product information can be improved based on dynamic linkage of heterogeneous knowledge representations. We therefore introduce a conceptual model of dialogue interaction based on multiple knowledge resources for in-store shopping situations and empirically test its utility with end-users.
\end{abstract}

Keywords: heterogeneous knowledge resources, dynamic linkage, dialogue interaction, ontology, empirical study.

\section{Introduction}

What if you could find a product that directly matches your personal preferences by posing a simple question on your mobile device? Today, customers in bricks-and-mortar stores often lack access to helpful product information from the Web, such as product manuals, user and professional reviews or feature comparisons. On the other hand, those customers that have in-store Web access by their mobile device may suffer from information overload due to the sheer quantity of product information available. We therefore investigate how to enable customer's access to comprehensive, helpful product information in physical shopping environments. In this context, we further study the filtering and intuitive presentation of such product information in form of dialogue interaction between customer and product. The use of product-centered dialogue systems in physical shopping environments enables an improved filtering and presentation of relevant product information [1] and thus satisfy the communication needs of customers as intuitive as possible. To realize such natural language communication between customers and physical products, dialogue systems and comprehensive knowledge representations are necessary [2]. But, knowledge resources around products suffer from heterogeneous nature and diverse semantics, e.g., product descriptions by several manufacturers, user and professional reviews on the web, explanations of product features by diverse providers, pricing and bundling information of specific portals. Here, we assume that product-centered 
dialogue interaction requires bundling of these product-related knowledge resources and their dynamic linkage at a specific "hot spot" for instance a web service. In this paper, we therefore introduce a conceptual model of dynamic linkage of product-related knowledge resources within dialogue interaction in instore shopping situations. By means of the resulting dialogue system prototype, we evaluate the utility of dialogue interaction based on heterogeneous knowledge resources from an end-user perspective. The focus of this contribution lies on the bundling of diverse heterogeneous knowledge resources, e.g., digital product descriptions, at a specific "hot spot" that enables not only standardized access to different types of information but also the dynamic linkage of these resources within in-store dialogue interaction. The rest of this paper is organized as follows. We will discuss related work in Sec. 2. Afterwards, our motivation will be illustrated by an example. In Sec. 4, the approach of bundling diverse heterogeneous knowledge resources is described with a focus on digital product descriptions. We then present our model of dynamic linkage of product-related knowledge resources within dialogue interaction for in-store shopping situations (Sec. 5). Finally, we show and evaluate an implementation of the model from an end-user perspective in Sec. 6, summarize our results and provide an outlook on future work (Sec. 7).

\section{Related Work}

In our work, dialogue interaction between customers and physical products in in-store shopping environments refers to the application of dialogue systems in physical environments and the usage of ontologies as knowledge representations. Dialogue systems provide the opportunity to interact with a system similar to human-human communication [3]. They can be divided into two basic types: dialogue grammars and frames as well as plan-based and collaborative systems. Dialogue grammars identify and represent surface patterns of dialogue or speech acts. Frame-based approaches extend grammars regarding their flexibility. Plan-based and collaborative systems assume that humans communicate to achieve goals and thus, focus on intentional structures [4]. When designing Natural Language Processing (NLP) modules, there are two extremes: full natural language processing or fixed linguistic question templates. A sweet spot between the two extremes is to constrain natural language in order to create a formal, user-friendly query language [5] or a controlled language for posing questions [6. There are diverse examples for current dialogue systems, for instance SmartKom - a multimodal dialogue system that combines speech, gesture and mimics input [7] as well as DELFOS, an dialogue manager system that enables the integration of OWL ontologies as external knowledge resources for dialogue systems [8]. The combination of NLP and ontologies facilitates the development of novel dialogue systems that use ontologies as a core knowledge component regarding linguistic and non-linguistic knowledge representations. In our case, product information as part of the non-linguistic knowledge base plays an important role. The effective handling of this heterogeneous product knowledge 
distributed among various steps in the product lifecycle has become essential [9. Meanwhile, there are several ontology-based developments that address the description of products. For instance, Product Design Ontology (AIM@SHAPE project) focuses on the formalization of knowledge concerning processes, tools and shapes during product development whereas other ontologies as GoodRelations [10] and SearchMonkey Product (by Yahoo!) are used to annotate digital products and service offerings on the web. An ontology within NLP constitutes the conceptualized description of the domain of the dialogue system [1]. We assume that NLP benefits from the appliance of semantic knowledge representations as well as semantic technologies, e.g., SWRL, in general. In the current work, dialogue interaction requires the generation of situation-specific questions and answers on run-time. Multiple pieces of information have to be combined while answering questions that are not anticipated at the time of system construction [12. The important role of the combination of language technologies, ontology engineering and machine learning is also described by Buitelaar et al. [13. The semantic web technologies and standards will be used for the specification of web-based, standardized language resources. However, building ontologies in the first place requires experienced knowledge engineers [12. The linkage of multiple ontology-based knowledge resources pose a challenge concerning the combination of knowledge of different resources and the answering of queries by considering multiple resources [14]. On the other hand, the effort of building up the knowledge base of the dialogue system can be decreased or sourced out. Furthermore, the coverage of dialogue systems is extended by integrating knowledge of multiple resources as it will be described in the current work 1514.

\section{Motivation}

The motivation for dialogue interaction in in-store shopping situations shall be described by a futuristic example of a sales talk. A customer enters a drugstore because she searches for a whitening toothpaste. She carries a smart phone that is also used to request additional information about products. She wants to take a look at the toothpastes that are right for her. She scans the barcode of a toothpaste with her smart phone to identify the product and poses the following question: "Which whitening toothpastes are available?" The toothpaste answers via mobile device: "There are three whitening toothpastes available: StarLight, Smile and WhiteSky. You can find it on the second floor. If you prefer, somebody will get it for you." Customer: "Yes, please." Toothpaste: "Are you interested in a video clip that explains the application of toothpaste StarLight?" Customer: "Why not!" A corresponding clip is shown on a display nearby. Customer: "Very good. Which mouthwashes fit to this toothpaste?" Toothpaste: "The best options are these two." Both are shown on her mobile device. Toothpaste: "If you buy this toothpaste and one of these mouthwashes you will get a $5 \%$ discount." Customer: "That's a good deal." The example has shown complex relationships between customers, manufacturers, products and product-related knowledge. We will depict these relationships in the next section. 


\section{Handling Clouds of Product-Centered Knowledge Resources}

In shopping environments, product-related knowledge is retrieved from different sources that use different semantics. Primarily, we speak about digital product descriptions that represent the informational basis to realize a customer-product dialogue. Currently, physical products are described non-standardized or standardized in terms of static databases (e.g., STEP ISO 10303 [16]) or XML structures (e.g., BMEcat: bmecat.org). Furthermore, product-centered knowledge resources also cover comparisons of products as well as their features. On the one hand, this information is provided by single manufacturer web sites exclusively considering their own products, e.g., Apple, Dell. On the other hand, consumer portals, e.g. Ciao! (ciao.co.uk), allocates comparisons of products manufactured by different companies. Further product-centered knowledge types are definitions or explanations of product features. Regarding the complexity of physical products, some features require explanations to enable customers to make confident purchase decisions. Currently, such explanations are available via diverse websites of manufacturers, search engines such as Google or online encyclopedias such as Wikipedia. In addition, the example in Sec. 3 shows the integration of pricing and product bundling information within the customer-product dialogue. Knowledge about matching products is provided by manufacturers exclusively concerning their own assortment as well as by shopping portals, e.g., Amazon, based on collaborative filtering mechanisms according to the principle "customers who bought this item also bought [...]". Pricing information on the web rarely exceed the scope of comparisons between retailing portals, for instance provided by ConsumerSearch (consumersearch.com). However, dynamic prices or discount bargains against dynamic parameters like customer type, current situation or inventory are not available. Finally, the aforementioned example presents a natural language dialogue between customer and product. Such dialogue interaction requires question-answering structures represented by linguistic knowledge resources. Currently, such linguistic knowledge resources are not freely accessible on the web. This short overview points out that there is a cloud of product-centered knowledge resources on the web, all of different semantics and formats. How can these "cloudy" information structures be integrated into a purposeful customer-product dialogue? In the following subsections, we will elaborate our approach for bundling such heterogeneous knowledge resources at a specific "hot spot" that enables standardized access to these different types of information. At this point, we focus on the handling of different formats of digital product descriptions as they represent essential product knowledge of each physical product. Finally, digital product descriptions build the basis for the calculation of dynamic prices [17] as well as product bundling results [18].

\subsection{Digital Product Information in Physical Environments}

As mentioned before, current physical products are mainly described in terms of static databases (e.g., eCl@ss: eclass-online.com) or XML structures (e.g., 


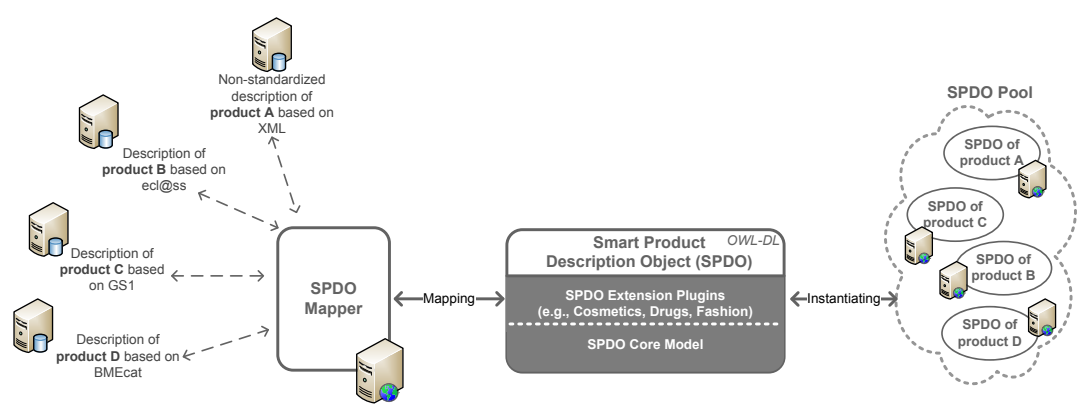

Fig. 1. Mapping of product descriptions of different formats into SPDO structure

BMEcat). Modeling enterprises or business processes is generally sophisticated (e.g., Business Process Modeling Notation: bpmn.org), but the description of products rarely exceeds the scope of classification. Furthermore, the effort in maintenance and extension of such product descriptions is high. So, product information is often incomplete or out-of-date. As product descriptions of diverse physical products in shopping environments serve as basis for our customerproduct dialogue, we have to handle the different formats of these descriptions (cf. Fig,1). We developed a mapping module that enables the automatic mapping of diverse XML-based product description formats into a standardized semantic product description structure - Smart Product Description Object (SPDO) - and vice versa. The web-based SPDO mapping interface is able to retrieve initial product description data from diverse servers, e.g., repositories of manufacturers. Afterwards, each product description is mapped automatically onto the core model of the SPDO structure. In case of imprecise relations between the concepts of the initial product description and SPDO structure, the web-based mapper interface allows the manual arrangement of concepts by the user. SPDO represents semantic and dynamic product information. It is a product ontology for physical products in in-store shopping environments [19]. As shown in Fig. 1 SPDO consists of a core mode 1 and SPDO specific extension plugins both formalized in OWL-DL. The further covers prototypical aspects of consumer products which is all domain-independent information of the product itself, e.g., name, color, price, manufacturer etc. Domain-specific conceptualizations, more precisely product information regarding specific product domains, can be imported into SPDO as extension plugins, for instance cosmetic 2 , drugs or fashion plugins. While mapping the original description of a physical product to SPDO structure, an instance of SPDO is generated. That is, each product description is transformed into one instantiated SPDO file formalized in OWL-DL (SPDO Pool)(cf. Fig 1). When we consider our in-store shopping environment, we can say that each physical product is then described by one SPDO instance. At the beginning of this subsection, we mentioned that product descriptions are

\footnotetext{
1 http://im.dm.hs-furtwangen.de/ontologies/spdo/2010b/SPDO.owl

2 http://im.dm.hs-furtwangen.de/ontologies/spdo/2010b/SPDO_Cosmetics.owl
} 
often incomplete or out-of-date because of high effort in maintenance and extension of such data. In order to address this issue, SPDO enables dynamic and automatic data extension and maintenance. Underspecified relations or concepts can be completed automatically with reasoning mechanisms. Therefore, product information based on SPDO will be extended by application of rules. A repository of web-based rules (SWRL ${ }^{3}$ ) enables the generation and integration of statements about alternative or matching products into SPDO instances, more precisely the processing of specific rules combines product descriptions automatically in real-time according to parameters that can be configured dynamically, for instance "color A fits to color B". As a result, an advanced and automatic processing in terms of updates and extensions is possible that forces the emergence of dynamically changing product networks.

\subsection{Bundling the Product-Centered Knowledge Cloud}

The network of SPDOs is one part of the product-centered knowledge cloud that needs to be processed to realize an intuitive representation of comprehensive product information in form of dialogue interaction. Fig. 2 shows our approach for bundling heterogeneous knowledge resources at a "hot spot" to enable standardized access to different types of product-centered knowledge. Besides SPDO Pool, we assume that following heterogeneous knowledge resources have to be bundled at a "hot spot" (cf. top layer in Fig. 2):

- Product-centered knowledge requested by external services is used to extend SPDOs, e.g., integration of explanations of specific product features or product reviews. Furthermore, external services like accessible thesauri or search engines are requested for linguistic information to extend the lexicon of the linguistic knowledge representation, e.g., adequate articles, plural forms of nouns etc.

- Currently, linguistic knowledge representations that match our needs regarding dialogue interaction in in-store shopping environments are not freely accessible on the web. We developed a light-weight linguistic knowledge representation formalized in OWL-DL that represents the structural backbone of dialogue interaction between customers and physical products. This knowledge resource will be elaborated in detail in the next section.

- The repository of web-based rules covers rules regarding matching or alternative products as well as rules concerning dynamic bundle prices and discounts. Rules are applied on SPDO Pool and provide results that are integrated in dialogue interaction.

- Sales and inventory figures of manufacturers or retailers represent large economic data resources that should be processed within a dialogue between customer and product. So, retailers are able to offer slow sellers in the context of matching products or allow dynamic discounts dependent on sales figures.

${ }^{3}$ Semantic Web Rule Language - http://www.w3.org/Submission/SWRL/ 


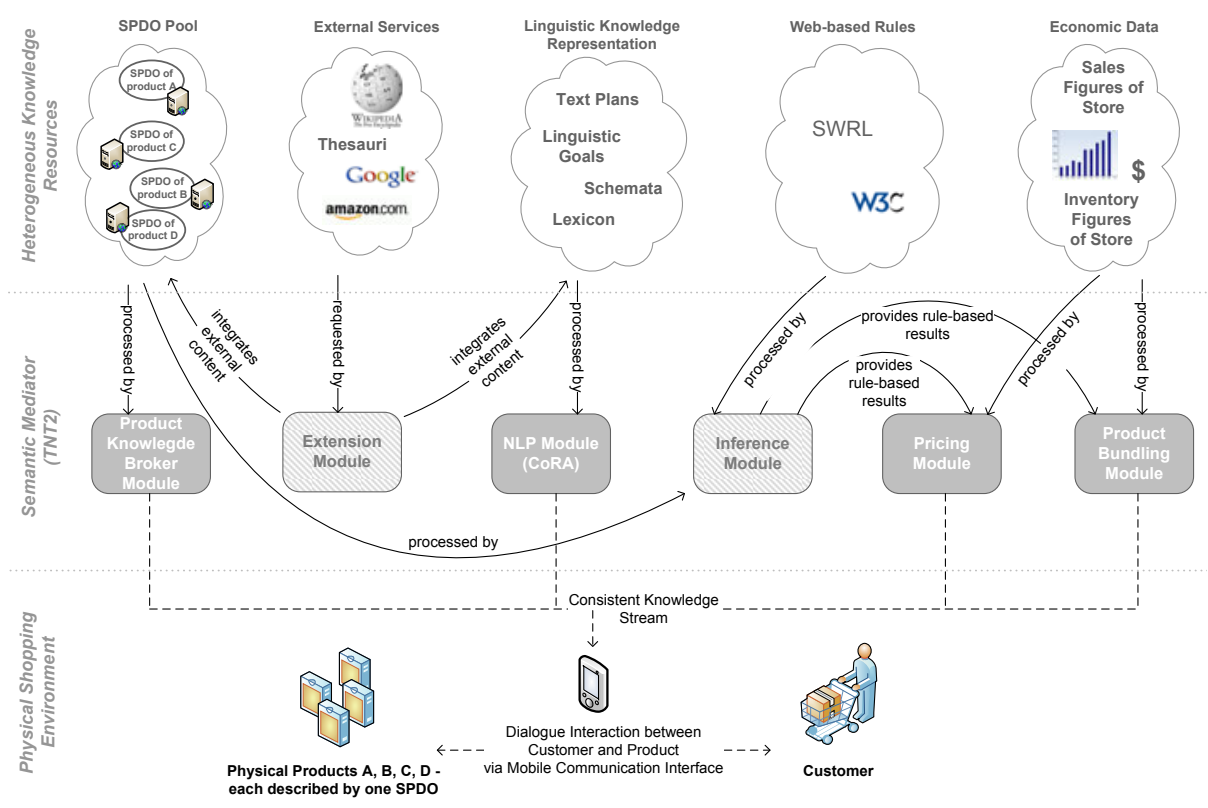

Fig. 2. Approach of bundling product-centered knowledge resources

These heterogeneous knowledge resources are bundled by a semantic mediator (cf. middle layer of Fig. 2) that represents the "hot spot" for generating a consistent knowledge stream based on resources of different semantics and formats. The semantic mediator consists of several modules that are responsible for requesting and processing diverse knowledge resources as well as allocating preliminary results. We discern internal (marked light grey) and interface modules (marked dark grey) (cf. Fig. 2). Internal modules generate preliminary results and forward these results to interface modules which directly contribute to the consistent knowledge stream. The semantic mediator contains two internal modules: Extension Module and Inference Module. The Extension Module requests knowledge from external services, e.g. definitions of product features via Wikipedia, and integrates this content into SPDOs or the linguistic knowledge representation. The second internal module - the Inference Module processes rules of the web-based rule repository and offers rule-based results to the Pricing and Product Bundling Module. Furthermore, Fig. 2] shows the following interface modules:

- Product Knowledge Broker Module processes product information stored in the SPDO Pool and induct this knowledge into the consistent knowledge stream.

- NLP Module processes the linguistic knowledge representation and allocates linguistic question-answer structures to realize dialogue interaction. 
- Pricing Module receives rule-based results from internal Inference Module as well as economic data and therefore calculates dynamic prices.

- Product Bundling Module processes sales and inventory figures of economic data as much as the Pricing Module. By merging economic data with rulebased results, matching or alternative product bundles are generated.

All described interface modules induct their processing results into the consistent knowledge stream whereas the semantic mediator coordinates linkage of the diverse results as well as alignment of knowledge offerings with the needs of the ongoing customer-product dialogue. The bottom layer of Fig. 2 shows the physical shopping environment consisting of customer, physical products and a mobile communication interface, e.g., the customer's mobile. All physical products are described by one SPDO instance, respectively. The mobile communication interface enables the dialogue interaction between customer and product. It represents an access point to the product-centered knowledge cloud and enables the customer to construct natural language questions term-by-term via choosing questions segments (written mode). Additionally, the customer is able to pose her question verbally (spoken mode). Afterwards, the mobile communication interface presents the generated answers with text and images. On technical level, the semantic mediator is implemented in TNT2 20] - an OSGi (osgi.org) based middleware for mobile recommendation agents in physical environments. The NLP Module is realized by the Conversational Recommendation Agent (CoRA) 21 that constitutes an OSGi plugin of TNT2. Furthermore, the client of the mobile communication interface was exemplarily developed on an Android (android.com) based mobile phone.

\section{Linkage of Heterogeneous Knowledge Resources for Dialogue Interaction}

Now, we know how to bundle heterogeneous knowledge resources based on a semantic mediator. But how can we dynamically merge these knowledge resources for the generation of answers of a dialogue interaction system? We want to generate situation-specific questions and answers for a dialogue at run-time that means questions and answers depend on concrete physical products in physical shopping situations with a specific context. These generation processes rely on the dynamic integration of heterogeneous knowledge resources. In detail, for answering queries by the user multiple external information resources are considered automatically. The integration of these external resources decrease the effort of building up knowledge representations for dialogue systems. In Fig. 3 . we present a model of dynamic linkage of heterogeneous knowledge resources for dialogue interaction. In this contribution, we focus on the linkage of product information from SPDO Pool with linguistic structures of the linguistic knowledge representation, because the NLP and Product Knowledge Broker module constitute the conceptual basis for dialogue interaction. The right part of Fig. 3 shows the conceptual design of the linguistic knowledge representation that represents the linguistic structures of the NLP approach [21. The representation 


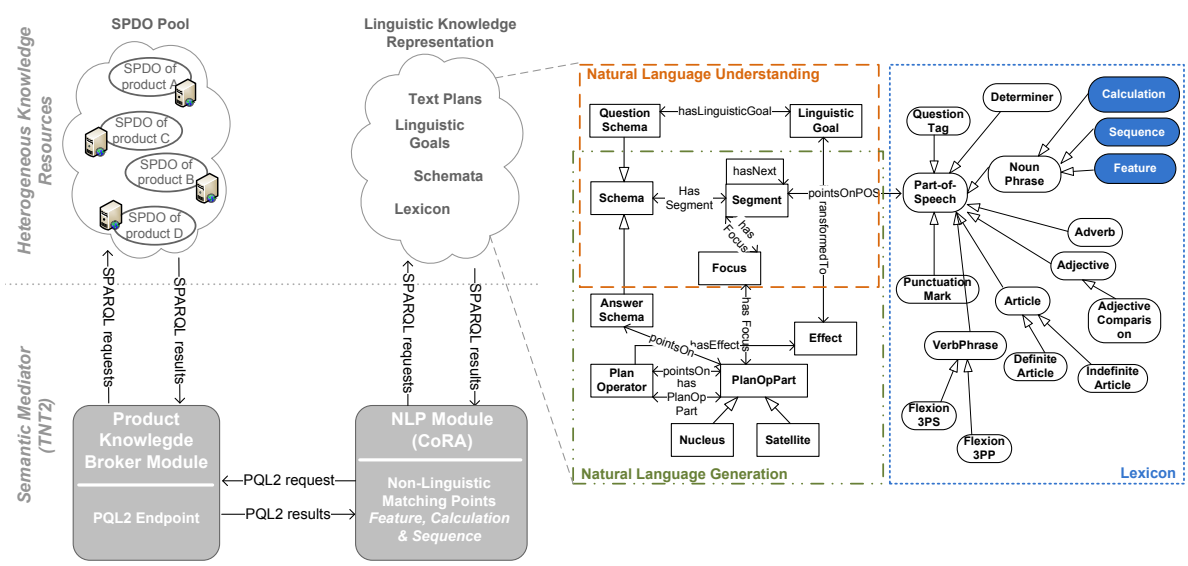

Fig. 3. Model of linkage of heterogeneous knowledge resources within dialogue interaction

was modeled based on an analysis of a German speech corpus of sales conversations and consulting talks concerning consumer electronics at a trade fair. After having transcribed and analyzed the corpus, we derived question structures of these purchase conversations. The linguistic representation can be subdivided into three parts: Natural Language Understanding (NLU), Natural Language Generation (NLG) and lexicon. NLU covers generic schemata of questions that are posed within the shopping domain. A semantic tree of questions is spanned based on question segments that are filled by words or phrases of the lexicon. An example of question schemata is listed as linguistic skeleton in Tab. 1. Similar to NLU, NLG represents generic linguistic structures of text plans that consists of answer schemata, which respond to the aforementioned questions. Examples of answer schemata are listed as linguistic skeletons in Tab. 1. Segments of question and answer schemata are filled by the German lexicon whose items base on the Penn Treebank Tagset 22]. As shown in Fig. 3, the lexicon contains three specific sub concepts of noun phrase: Calculation, Feature and Sequence. These concepts represent Non-Linguistic Matching Points that operate as gates for linking product-domain-specific information with the linguistic skeleton of questions or answers. Calculation, Feature and Sequence contain several subtypes that represent question and answer segments with specific functions (cf. Tab. (2). While processing the generic linguistic structure (cf. Tab. 1, Col. 2), the NLP Module detects noun phrases of type Calculation, Feature or Sequence and triggers the Product Knowledge Broker Module that allocates a PQL2 endpoint (cf. Fig. 3). A PQL2 request concerning the detected subtype of Non-Linguistic Matching Point, e.g., "ProductCategoryValue" is sent to the PQL2 endpoint by the NLP Module (cf. Tab. 1. Col. 3). PQL2 is a high-level semantic query language that allows to request pools of multiple ontologies via a light-weight 
Table 1. Examples of Question-Answer Flows

\begin{tabular}{|c|c|c|c|}
\hline $\begin{array}{l}\text { Type of Non- } \\
\text { Linguistic } \\
\text { Matching } \\
\text { Point }\end{array}$ & $\begin{array}{l}\text { Examples of Lin- } \\
\text { guistic Skeleton }\end{array}$ & $\begin{array}{l}\text { PQL2 } \\
\text { query }\end{array}$ & $\begin{array}{l}\text { Examples of final } \\
\text { output }\end{array}$ \\
\hline $\begin{array}{l}\text { (Feature) Pro- } \\
\text { ductCategory- } \\
\text { Value }\end{array}$ & $\begin{array}{l}<\text { Which }>\text { [Product- } \\
\text { CategoryValue] } \\
<\text { are available }> \\
<\text { with }>\quad \text { Product- } \\
\text { Property] [Product- } \\
\text { PropertyValue]? }\end{array}$ & $\begin{array}{l}\text { SPDO* } \\
<\text { pql2:product- } \\
\text { category }> \\
\text { <pql2:plural }> \\
\text { ?value }\end{array}$ & $\begin{array}{l}\text { Which bodylotions } \\
\text { are available with }[\ldots] \\
\text { Which toothpastes } \\
\text { are available with }[\ldots]\end{array}$ \\
\hline $\begin{array}{l}\text { (Sequence) } \\
\text { ProductProp- } \\
\text { ertyValueSEQ }\end{array}$ & $\begin{array}{l}\text { [ProductCategoryValue] } \\
<\text { is available in }> \\
\text { [NumberOf] [Pro- } \\
\text { ductProperty]: } \\
\text { [ProductProp- } \\
\text { ertyValueSEQ] }\end{array}$ & $\begin{array}{l}\text { SPDO } \\
<\text { pql2:product- } \\
\text { property[property] }> \\
<\text { pql2:seq }>\text { ?value }\end{array}$ & $\begin{array}{l}\text { Jackets are available } \\
\text { in four colors: White, } \\
\text { Black, Grey and } \\
\text { Blue. } \\
\text { Toothpastes are avail- } \\
\text { able in three flavors: } \\
\text { Mint, Cherry and } \\
\text { Orange. }\end{array}$ \\
\hline $\begin{array}{l}\text { (Calculation) } \\
\text { BundlePrice }\end{array}$ & $\begin{array}{l}\text { [ProductValueSEQ] } \\
<\text { cost }>\quad<\text { as a } \\
\text { bundle only }> \\
\text { [BundlePrice]. }\end{array}$ & $\begin{array}{l}\text { SPDO* } \\
<\text { pql2:bundle- } \\
\text { price(<pql2:product- } \\
\text { value[product- } \\
\text { value] }><\text { pql2:seq }>\text { ) }> \\
\text { ?value }\end{array}$ & $\begin{array}{l}\text { iPod nano and iPad } \\
\text { cost as a bundle only } \\
\mathbf{8 5 0} \text { USD. } \\
\text { Shoes Sunshine and } \\
\text { t-shirt Summer cost } \\
\text { as a bundle only } \mathbf{5 5} \\
\text { USD. }\end{array}$ \\
\hline
\end{tabular}

Table 2. Subtypes of Non-Linguistic Matching Points

\begin{tabular}{lll}
\hline Feature & Sequence & Calculation \\
\hline ProductCategoryValue & ProductPropertySEQ & BundlePrice \\
ProductCategoryValuePlural & ProductPropertyValueSEQ & Discount \\
ProductProperty & ProductValueSEQ & NumberOf \\
ProductPropertyPlural & ProductCategorySEQ & PriceAverage \\
ProductPropertyValue & ProductProperty - PropertyValueSEQ & PriceThreshold \\
ProductValue & & \\
\hline
\end{tabular}

endpoint with simple queries that will be internally transformed in an SPARQI 4 request. Below, an exemplary PQL2 query of Tab. 1 is elaborated:

$$
\text { SPDO <pq12:product-property[property]> 〈pq12:seq> ?value }
$$

This PQL2 query represents the request of a sequence $(<\mathrm{pql2}: \mathrm{seq}\rangle)$ of values of a specific property of one product (SPDO), e.g., <pq12: product-property [color] >. In contrast, the term SPDO* expresses that the whole pool of SPDOs is requested (cf. Tab. 1). In summary, PQL2 offers the following features:

- PQL2 analyzes the ontological structure of the ontologies in target concerning their concepts and relation. Then, PQL2 offers specific requesting

4 http://www.w3.org/TR/rdf-sparql-query/ 
items according to the ontological structure, e.g., <pql2:product-category $>$ $<$ pql2:plural $>$. This means that external modules that use PQL2 need no knowledge about the constitution of the ontological knowledge base they request.

- PQL2 allows to request data of multiple ontologies, e.g., SPDO Pool, via a single simplified PQL2 request (cf. Fig. 3).

- PQL2 enables the integration of semantic statements into the ontological structures, e.g., based on information of external services or rule-based results.

- With PQL2 other modules of the semantic mediator can be requested such as Pricing or Product Bundling modules.

After receiving the PQL2 request by the NLP Module, the Product Knowledge Broker Module transforms the request into one or several SPARQL queries to request the SPDO Pool or further modules. The results are sent to the NLP Module that inserts them into the linguistic skeleton to generate the final output (cf. Tab. 1, Col. 4). The linkage of the linguistic knowledge representation and SPDO pool via PQL2 queries is shown by means of an example consisting of question and answer in Fig. 4. The red labeled words and phrases are filled from on PQL2 queries (the right part of Fig. 4). Imagine, the user wants to know which fragrances are available for the product Sunshine Bodylotion. While composing the desired question, she selects the question segment "fragrances" from a list of product properties (ProductProperySEQ) that are available for the product.

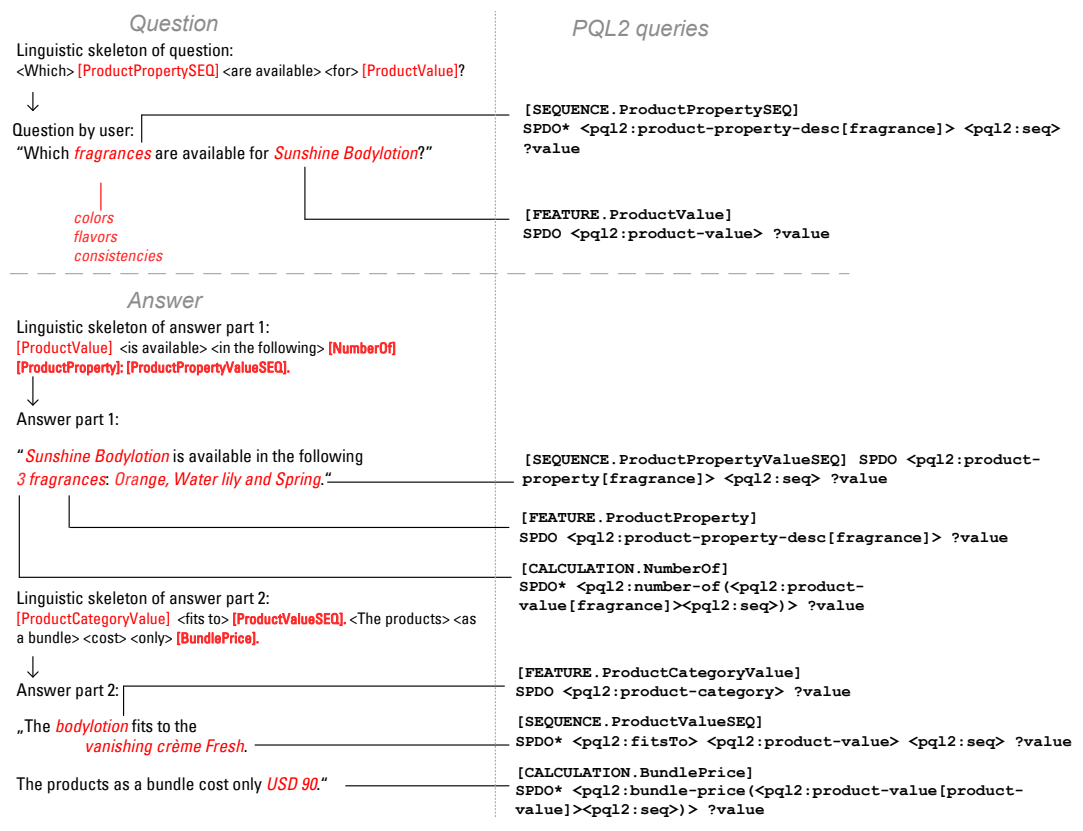

Fig. 4. Example of Question-Answering regarding PQL2 requests 
In this context, further possible properties would be Color, Flavor or Consistency. The right part of the figure shows the PQL2 queries that are processed to enrich the linguistic skeleton. The question "Which fragrances are available for Sunshine Bodylotion?" is responded by an answer consisting of two parts. First, "Sunshine Bodylotion is available in the following 3 fragrances: Orange, Water lily and Spring." The Sequence (ProductPropertyValueSEQ) of fragrance values as well as the name of the property "fragrance" (ProductProperty) and the calculation of the number of fragrances available (NumberOf) is generated by a PQL2 request. The second part of the answer presents a sequence of matching products (ProductValueSEQ) gained via a PQL2 request that triggers the Product Bundling Module whereas the corresponding bundle price (BundlePrice) is filled with results of the Pricing Module.

\section{Utility of Ontology-Based Dialogue Interaction}

Having described the model for ontology-based dialogue interaction above, we now test its utility for in-store shopping situations from an end-user perspective. For this purpose, we implemented a Conversational Recommendation Agent (CoRA) that is derived from the proposed model and provides a communicative interface between consumers and physical products at the point of sale. Technically, CoRA is an OSGi plugin of TNT2. The CoRA client is implemented on a mobile phone. It allows consumers to identify a product by barcode via the phone's built-in camera and then to ask for product information as shown in Fig. 5 to 7 . In the current work, utility is defined as the degree to which ontology-based dialogue interaction is adequate for end-users to request product information in in-store shopping situations. Accordingly, CoRA is an implementation of this concept. In order to evaluate the utility of CoRA, we use the following constructs from information systems research: perceived ease of use and perceived enjoyment [23], relative advantage of CoRA compared (1) to static product information such as printed product labels, and (2) to a sales talk [24] and finally, intention to use CoRA 25. An experiment was conducted, in which each subject was asked to use CoRA to request information of several cosmetic products. The subjects had to ask the following questions to get used to CoRA and to be able to evaluate it afterwards: What is the price of the product? Which products fit to this product? Are there alternative products available? Are there less expensive products of this product category available? What is the average price of this product category? All of these questions were derived from in-store sales talks and thus, are relevant in a shopping situation. With the CoRA client, each subject was able to construct the questions term-by-term whereas CoRA suggested only those terms from which the questions could be constructed meaningfully (Fig. 6). The subjects had therefore not to type in the questions manually but were only asked to chose terms they were interested in by tapping with their finger (e.g., Which - products - fit...). During the session of 30 minutes, further guidance was provided when a subject asked for additional help with CoRA. Then, in the second part of the experiment, the subjects were 


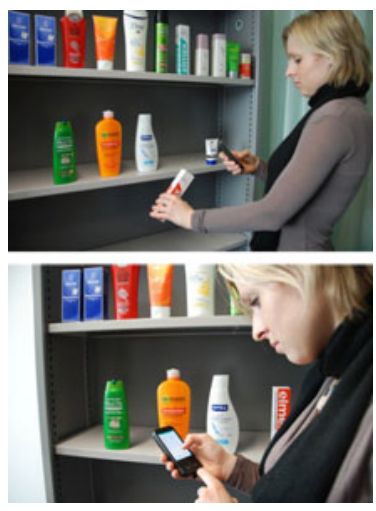

Fig. 5. Subject with CoRA in front of a product shelf

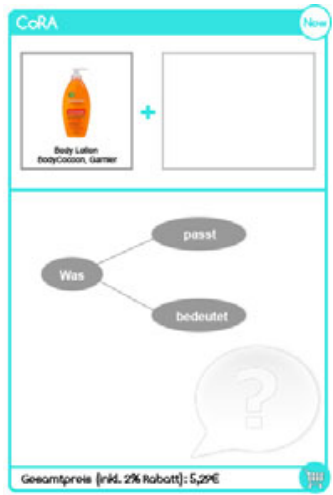

Fig. 6. Step-by-step composition of a question

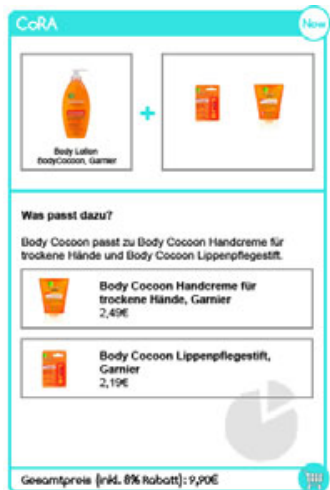

Fig. 7. Presentation of the answer

asked to rate questionnaire items with regard to the theoretical constructs described above. Consistent with prior research, we adopted 7-point Likert scales that range from strongly disagree (1) to strongly agree (7).

All in all, 19 female and 37 male subjects studying at a business university participated in the experiment. Their age ranged from 20 to $24(n=34), 25$ to $29(\mathrm{n}=13)$. The seven remaining subjects were above 30 . We employed onesample t-tests with a neutral test value of 4 to indicate whether the results are significantly positive or negative resulting in high or low utility scores for CoRA. The descriptive statistics and the results of the one-sample t-tests are shown in Tab. 3. All multi-item research constructs were reliable as Cronbach's Alpha lies above the recommended value of .70 [26]. The one-sample t-tests indicate that almost all constructs were perceived positive at the highest level of significance

Table 3. Descriptive statistics and results of the one-sample t-test for the 54 participants; Note: $\mathrm{SD}=$ standard deviation

\begin{tabular}{lcccccc}
\hline Construct & Items & Alpha & Mean & SD & p-value & Interpretation \\
\hline $\begin{array}{l}\text { Perceived ease of use of } \\
\text { CoRA }\end{array}$ & 3 & .761 & 5.70 & 0.87 & $<.001$ & CoRA was easy to use \\
\hline $\begin{array}{l}\text { Perceived enjoyment of } \\
\text { CoRA }\end{array}$ & 3 & .838 & 5.31 & 1.09 & $<.001$ & $\begin{array}{l}\text { CoRA has made fun dur- } \\
\text { ing usage }\end{array}$ \\
\hline $\begin{array}{l}\text { Perceived relative ad- } \\
\text { vantage of CoRA when } \\
\text { compared to static infor- } \\
\text { mation }\end{array}$ & 3 & .748 & 4.59 & 1.26 & $<.01$ & $\begin{array}{l}\text { CoRA was perceived } \\
\text { better relative to static } \\
\text { product information }\end{array}$ \\
\hline $\begin{array}{l}\text { Perceived relative ad- } \\
\text { vantage of CoRA when } \\
\text { compared to a sales talk }\end{array}$ & 3 & .802 & 4.03 & 1.25 & $>.05$ & $\begin{array}{l}\text { CoRA was neither per- } \\
\text { ceived better nor worse } \\
\text { than a sales talk }\end{array}$ \\
\hline \begin{tabular}{l} 
Intention to use CoRA \\
\hline
\end{tabular} & 1 & n/a & 5.59 & 1.39 & $<.001$ & $\begin{array}{l}\text { The participants would } \\
\text { intend to use CoRA }\end{array}$ \\
\hline
\end{tabular}


at .001, which supports the utility of CoRA for product information acquisition in in-store shopping situations. Only when compared to a sales talk, CoRA shows no significant relative advantage but also no significant disadvantage. We therefore assume that CoRA is comparable to a sales talk, which does not only strengthen its utility for consumers but also for retailers that may offer such a mobile application in addition to sales personnel. Although this experiment and its results are limited to the domain of cosmetics and are based on a relatively small sample obtained from a university, the results are promising and may apply for other consumer products as well. We therefore will conduct further experiments to validate the positive results for other product domains in field experiments.

\section{Conclusion and Future Work}

Dialogue interaction between customers and products encompasses the capability for improved filtering and presentation of relevant product information in in-store shopping situations. Thus, information needs of customers can be addressed more intuitive. In order to realize such interaction, dialogue systems and comprehensive knowledge representations are necessary such as product information, linguistic representations and user reviews. Because of their heterogeneous nature and diverse semantics, these product-related knowledge resources have to be bundled and linked to enable standardized information access. In this article, we have introduced a conceptual model of dynamic linkage of product-related knowledge resources for dialogue interaction in in-store shopping situations. It was shown how multiple heterogeneous knowledge resources are bundled by a $S e$ mantic Mediator that enables standardized access to different types of productcentered knowledge resources. Especially, the mapping of product descriptions of diverse formats, e.g., BMEcat, into a semantic product description structure is elaborated. This standardized ontological product description of physical products is dynamically updated and extended via SWRL. Afterwards, we have described our model of dynamic linkage of these bundled knowledge resources, i.e. the linkage of an ontological linguistic knowledge base of the dialogue system with a pool of product descriptions. We apply ontologies as non-linguistic and linguistic core knowledge components of our dialogue system. The semantic knowledge is merged automatically with external non-semantic contents. This is enabled by a high level semantic query language that maps ontological data into Java structures and thus allows object-oriented querying of semantic data. With a prototype of the dialogue system, we have then shown the utility of in-store dialogue interaction based on heterogeneous knowledge resources by an end-user study. In our future work, we will focus on three issues: (1) extension of the linkage model with more product-related knowledge resources, (2) enhancement and standardization of PQL2 functionality, and (3) augmentation of our linguistic resource by linking up further external services such as dbpedia.org or zemanta.com. 


\section{Acknowledgement}

We would like to thank Eva Blomqvist for her comprehensive advices concerning SPDO. This paper resulted from project 'Interactive Knowledge Stack (IKS)' (FP7 231527) co-funded by the European Commission.

\section{References}

1. Gurevych, I., Mühlhäuser, M.: Natural language processing for ambient intelligence. Künstliche Intelligenz/Special Issue: Ambient Intelligence und Künstliche Intelligenz (2), 10-16 (2007)

2. Sabou, M., Kantorovitch, J., Nikolov, A., Tokmakoff, A., Zhou, X., Motta, E.: Position paper on realizing smart products: Challenges for semantic web technologies. In: The 2nd International Workshop on Semantic Sensor Networks, collocated with ISWC 2009 (2009)

3. Bel-Enguix, G., Dediu, A.-H., Jimenez-Lopez, M.: A dialogue-based system for man-machine interaction. In: Conf. on Human System Interactions, pp. 141-146 (25-27, 2008)

4. Catizone, R., Wilks, Y., Worgan, S., Turunen, M.: Some background on dialogue management and conversational speech for dialogue systems. In: Wilks, Y., Catizone, R. (eds.) Computer, Speech and Language (2010) (special issue on dialogue)

5. Warren, H.D.D., Pereira, C.N.F.: An efficient easily adaptable system for interpreting natural language queries. Computational Linguistics (8), 110-122 (1982)

6. Clark, P., Chaw, S.Y., Barker, K., Chaudhri, V., Harrison, P., Fan, J., John, B., Porter, B., Spaulding, A., Thompson, J., Yeh, P.: Capturing and answering questions posed to a knowledge-based system. In: K-CAP 2007: Proc. of the 4th International Conf. on Knowledge Capture, pp. 63-70. ACM, New York (2007)

7. Alexandersson, J., Becker, T., Pfleger, N.: Overlay: The basic operation for discourse processing. In: Wahlster, W. (ed.) SmartKom: Foundations of Multimodal Dialogue Systems, pp. 255-267 (2006)

8. Perez, G., Amores, G., Manchon, P., Gonzalez, O.G.Y.J., Julietta, G.I.: Integrating owl ontologies with a dialogue manager. Technical report, CiteSeerX - Scientific Literature Digital Library and Search Engine (2006)

9. Chen, Y.J., Chen, Y.M., Chu, H.C.: Development of a mechanism for ontologybased product lifecycle knowledge integration. Expert Syst. Appl. 36(2), 2759-2779 (2009)

10. Hepp, M.: Goodrelations: An ontology for describing products and services offers on the web. In: Gangemi, A., Euzenat, J. (eds.) EKAW 2008. LNCS (LNAI), vol. 5268, pp. 329-346. Springer, Heidelberg (2008)

11. Ou, S., Pekar, V., Orasan, C., Spurk, C., Matteo, N.: Development and alignment of a domain-specific ontology for question answering. In: European Language Resources Association (ed.) Proc. of the Sixth International Language Resources and Evaluation (LREC 2008), Marrakech, Morocco (2008)

12. Clark, P., Thompson, J., Porter, B.: A knowledge-based approach to questionanswering. In: Proc. AAAI 1999 Fall Symposium on Question-Answering Systems, pp. 43-51 (1999)

13. Buitelaar, P., Declerck, T., Calzolari, N., Lenci, A.: Language resources and the semantic web. In: Proc. of the ELSNET/ENABLER Workshop (2003) 
14. Lopez, V., Uren, V., Motta, E., Pasin, M.: AquaLog: an ontology-driven question answering system for organizational semantic intranets. Web Semantics 5(2), 72 105 (2008)

15. Hayashi, Y., Declerck, T., Buitelaar, P., Monachini, M.: Ontologies for a global language infrastructure. In: Webster, J., Ide, N., Fang, A.C. (eds.) Proc. of the 1st International Conf. on Global Interoperability for Language Resources (ICGL 2008), Hong Kong, China, pp. 105-112 (2008)

16. Anderl, R., Trippner, D.: Step standard for the exchange of product model data. Technical report, STEP (2000)

17. Kowatsch, T., Maass, W.: Towards a framework for knowledge-based pricing services improving operational agility in the retail industry. In: D'Andrea, V., Gangadharan, G.R., Iannella, R., Weiss, M. (eds.) CEUR Workshop Proc., vol. 530 (2009)

18. Kowatsch, T., Maass, W., Filler, A., Janzen, S.: Knowledge-based bundling of smart products on a mobile recomendation agent. In: ICMB 2008: Proc. of the 7th International Conf. on Mobile Business, Washington, DC, USA, pp. 181-190. IEEE Computer Society, Los Alamitos (2008)

19. Maass, W., Janzen, S.: A pattern-based ontology building method for ambient environments. In: Blomqvist, E., Sandkuhl, K., Scharffe, F., Svatek, V. (eds.) Proc. of the Workshop on Ontology Patterns (WOP 2009), collocated with ISWC 2009, Washington D.C., vol. 516, CEUR Workshop Proc. (2009)

20. Maass, W., Filler, A.: Towards an infrastructure for semantically annotated physical products. In: Gesellschaft für Informatik e. V (ed.) Conf. Proc. Informatik 2006 (2006)

21. Janzen, S., Maass, W.: Ontology-based natural language processing for in-store shopping situations. In: Proc. of Third IEEE International Conf. on Semantic Computing (ICSC 2009), pp. 361-366. IEEE Computer Society, Los Alamitos (2009)

22. Marcus, M.P., Marcinkiewicz, M.A., Santorini, B.: Building a large annotated corpus of english: the penn treebank. Comput. Linguist. 19(2), 313-330 (1993)

23. Kamis, A., Koufaris, M., Stern, T.: Using an attribute-based decision support system for user-customized products online: An experimental investigation. MIS Quarterly 32(1), 159-177 (2008)

24. Moore, G., Benbasat, I.: Development of an instrument to measure the perceptions of adopting an information technology innovation. Information Systems Research 2, 173-191 (1991)

25. Davis, F.D.: Perceived usefulness, perceived ease of use, and user acceptance of information technology. MIS Quarterly 13(3), 319-339 (1989)

26. Nunnally, J.C.: Psychometric Theory. McGraw-Hill, New York (1967) 